\title{
Mineralização do carbono e do nitrogênio no solo após sucessivas aplicações de lodo de esgoto
}

\author{
Cristiano Alberto de Andrade ${ }^{(1)}$, Lívia Fernanda Mendonça Silva(2), Adriana Marlene Moreno Pires ${ }^{(1)}$ \\ e Aline Reneé Coscione(2)
}

\begin{abstract}
(1)Embrapa Meio Ambiente, Rodovia SP 340, Km 127,5, Caixa Postal69, CEP 13820-000 Jaguariúna, SP. E-mail: cristiano.andrade@embrapa.br, adriana.pires@embrapa.br (2)Instituto Agronômico, Laboratório de Qualidade do Solo, Avenida Barão de Itapura, no 1.481, Caixa Postal 28, CEP 13012-970 Campinas, SP. E-mail: liviaferms@bol.com.br, aline@iac.sp.gov.br
\end{abstract}

Resumo - O objetivo deste trabalho foi avaliar a dinâmica de mineralização do $\mathrm{C}$ e a capacidade do solo em fornecer $\mathrm{N}$ após sucessivas aplicações de lodo de esgoto, além de determinar a relação destas com a taxa de mineralização do lodo previamente aplicado. A área experimental recebeu, por sete anos, os seguintes tratamentos: $80 \mathrm{~kg} \mathrm{ha}^{-1}$ por ano de $\mathrm{N}$ mineral (L0); e 10 ou $20 \mathrm{Mg} \mathrm{ha}^{-1}$ por ano de lodo (L1 e L2, respectivamente). Amostras de solo $(0-20 \mathrm{~cm})$ foram retiradas um ano após a última aplicação dos tratamentos. As amostras receberam doses de lodo correspondentes a 0,120 e $240 \mathrm{~kg} \mathrm{ha}^{-1}$ de $\mathrm{N}$ e foram incubadas em laboratório por mais de 100 dias, tendo-se determinado o $\mathrm{C}-\mathrm{CO}_{2}$ liberado e o teor de $\mathrm{N}$ inorgânico no solo. A mineralização do $\mathrm{C}$ e do $\mathrm{N}$ foi mais influenciada pelo uso prévio do lodo do que pelas doses incubadas. A taxa de mineralização do nitrogênio (TMN) diminuiu de 16\%, em L0, para 11\%, em L1, e 8\% em L2. No entanto, essa redução não resultou em menor disponibilidade de $\mathrm{N}$ no sistema, pois houve incremento do $\mathrm{N}$ potencialmente mineralizável $\left(\mathrm{N}_{0}\right)$ em L1 e L2. Assim, a TMN não é um parâmetro adequado para cálculo da dose de lodo a ser aplicada em áreas previamente tratadas com o resíduo, pois subestima a capacidade do sistema em fornecer N. Neste caso, deve-se considerar o uso do $\mathrm{N}_{0}$.

Termos para indexação: fertilizante nitrogenado, matéria orgânica, nitrogênio potencialmente mineralizável, resíduo, taxa de mineralização do nitrogênio.

\section{Mineralization of carbon and nitrogen in soil after successive applications of sewage sludge}

\begin{abstract}
The objective of this work was to evaluate the dynamics of carbon mineralization and the soil capacity in providing nitrogen after successive applications of sewage sludge, as well as to determine their relation with the mineralization rate of the sludge previously applied. The experimental area received for seven years the following treatments: $80 \mathrm{~kg} \mathrm{ha}^{-1}$ per year of mineral $\mathrm{N}$ (L0); and 10 or $20 \mathrm{Mg} \mathrm{ha}^{-1}$ per year of sewage sludge (L1 and L2, respectively). Soil samples $(0-20 \mathrm{~cm})$ were taken one year after the last application of the treatments. Samples received sewage sludge doses corresponding to 0,120 , and $240 \mathrm{~kg} \mathrm{ha}^{-1}$ of N and were incubated in laboratory for more than 100 days, with determination of $\mathrm{C}-\mathrm{CO}_{2}$ emission and inorganic soil $\mathrm{N}$ content. The mineralization of $\mathrm{C}$ and $\mathrm{N}$ was more influenced by the previous use of sludge than by the incubated doses. The nitrogen mineralization rate (NMR) reduced from $16 \%$, on $\mathrm{L} 0$, to $11 \%$ on $\mathrm{L} 1$ and $8 \%$ on L2. However, this reduction did not result in lower $\mathrm{N}$ availability on the system, since there was an increase of the potentially mineralizable nitrogen $\left(\mathrm{N}_{0}\right)$ in $\mathrm{L} 1$ and L2. Therefore, the NMR is not an adequate parameter for calculating the sewage sludge dose to be applied on areas previously treated with the residue, since it underestimates the system capacity of providing $\mathrm{N}$. In this case, the use of $\mathrm{N}_{0}$ should be considered.
\end{abstract}

Index terms: nitrogen fertilizer, organic matter, potentially mineralizable nitrogen, waste, nitrogen mineralization rate.

\section{Introdução}

O uso agrícola do lodo de esgoto tem sido apontado como alternativa adequada para destinação final desse resíduo, uma vez que ele pode substituir total ou parcialmente o uso de fertilizantes minerais, principalmente os nitrogenados (Franco-Hernández et al., 2003; Bettiol \& Camargo, 2006).

A dinâmica de degradação da carga orgânica adicionada governa o potencial do resíduo em liberar 
N para o solo (Parker \& Sommers, 1983). Por esse motivo, um dos parâmetros avaliados quando se calcula a dose de lodo para fertilizações é a capacidade do resíduo em fornecer nitrogênio para as plantas.

A Resolução no 375 do Conama - norma federal que regulamenta o uso agrícola de lodo de esgoto (Conselho Nacional do Meio Ambiente, 2006) - indica que as doses devem ser calculadas de acordo com diferentes atributos do lodo (disponibilidade de N, alcalinidade e teor de metais pesados). A menor dose obtida nestes cálculos é a que deve ser utilizada na aplicação. $\mathrm{Na}$ maioria dos casos, a dose calculada com base na taxa de mineralização do nitrogênio (TMN) é a menor; portanto, essa taxa é o parâmetro mais frequentemente utilizado na recomendação de doses de lodo de esgoto.

A TMN pode ser determinada experimentalmente, mas, na maioria dos países, são utilizados valores pré-definidos pela legislação para cada processo de obtenção do lodo (tratamento do esgoto e processos adicionais). A Resolução no 375 do Conama adota os mesmos valores de TMN recomendados pela Agência Ambiental dos EUA (United States Environmental Protection Agency, 1993), que variam de 40\%, para lodos não digeridos, a $10 \%$, para lodos compostados. Neste ponto, cabe destacar que a mineralização do nitrogênio é influenciada por fatores climáticos (Paul et al., 2002) e que a adoção de valores obtidos em regiões temperadas pode resultar em erros na estimativa quando extrapolados para as condições brasileiras. Ao se assumir que estes valores sejam adequados para o cenário nacional, taxas inferiores a 50\% resultariam numa dose de lodo que contenha pelo menos o dobro da quantidade de $\mathrm{N}$ (total) demandada pela cultura. Portanto, fica evidente a necessidade de se levar em consideração o $\mathrm{N}$ potencialmente mineralizável proveniente de aplicações anteriores do resíduo, para o cálculo da próxima aplicação. No entanto, isso não está previsto na Resolução. No trabalho clássico de Pratt et al. (1973), discute-se a redução nos valores da TMN com o tempo, após a aplicação de diferentes resíduos orgânicos ao solo. No caso do lodo líquido, os autores estimaram a TMN em 35\% no primeiro ano, 10\% no segundo ano e $5 \%$ a partir do terceiro ano. Segundo os autores, a disponibilidade de $\mathrm{N}$ nos anos subsequentes à aplicação de lodo é função da TMN do material remanescente.

Dessa forma, o uso de doses fixas de lodo pode aumentar o $\mathrm{N}$ mineralizado com o tempo (Lindemann et al., 1988). Esta situação representa risco ambiental, pois o aumento gradual da capacidade do solo em fornecer N (Jin et al., 2011) deve, teoricamente, ser acompanhado da redução nas doses aplicadas, para prevenir o excesso de $\mathrm{N}$ inorgânico, a lixiviação de $\mathrm{NO}_{3}{ }^{-}$ para águas subterrâneas (Medaile et al., 1994; Oliveira et al., 2001; Dynia et al., 2006) e a emissão de $\mathrm{N}_{2} \mathrm{O}$ para a atmosfera (Chiaradia et al., 2009; Pitombo, 2011).

Trabalhos que avaliam a mineralização do $\mathrm{N}$ de lodos de esgoto após aplicação no solo são comuns; porém, os que avaliam áreas sujeitas a sucessivas aplicações do resíduo e estimam a quantidade mineralizável de $\mathrm{N}$ para cálculo das doses são escassos (Felipó \& Garau, 1987; Boyle \& Paul, 1989; Boeira, 2005; Hseu \& Huang, 2005; Boeira \& Maximiliano, 2009; Andrade et al., 2010; Gil et al., 2011). A quantificação do efeito residual do lodo e o estabelecimento da relação entre o $\mathrm{N}$ potencialmente mineralizável no solo e a nova dose para aplicação são caminhos que podem aprimorar o sistema de recomendação de lodo no Brasil (Conselho Nacional de Meio Ambiente, 2006).

O objetivo deste trabalho foi avaliar a dinâmica de mineralização do $\mathrm{C}$ e a capacidade do solo em fornecer $\mathrm{N}$ após sucessivas aplicações de lodo de esgoto, além de determinar a relação destas com a taxa de mineralização do lodo previamente aplicado.

\section{Material e Métodos}

Os estudos para avaliação da mineralização do $\mathrm{C}$ e do $\mathrm{N}$ foram conduzidos em laboratório, com uso de amostras de Latossolo Vermelho eutroférrico (Santos et al., 2006), de textura argilosa, coletadas em experimento de longa duração. Esse experimento utilizou fertilizante nitrogenado mineral e diferentes doses de lodo de esgoto, e foi realizado no Centro Experimental Central do Instituto Agronômico (IAC), em Campinas, SP. A área experimental foi estabelecida em 2001, com três tratamentos: L0, controle, sem uso de lodo de esgoto, mas com fertilização nitrogenada mineral ( $\left.80 \mathrm{~kg} \mathrm{ha}^{-1}\right)$; e L1 e L2, doses de lodo de esgoto calculadas para fornecimento de $80 \mathrm{e}$ $160 \mathrm{~kg} \mathrm{ha}^{-1}$ de $\mathrm{N}$, respectivamente. As aplicações do lodo ou do fertilizante nitrogenado mineral foram feitas anualmente, entre os anos agrícolas 2001/2002 e 2007/2008 (Galdos et al., 2004; De Maria et al., 2007, 2010; Pitombo, 2011; Bueno et al., 2011). Ao longo do experimento, o milho foi utilizado como cultivo de verão.

Pesq. agropec. bras., Brasília, v.48, n.5, p.536-544, maio 2013 DOI: $10.1590 / \mathrm{S} 0100-204 X 2013000500010$ 
Em 2001, a dose de lodo aplicada correspondeu a 10,8 e $21,6 \mathrm{Mg} \mathrm{ha}^{-1}$ (massa seca), e foi definida de acordo com a análise química do lodo, a necessidade de $\mathrm{N}$ da cultura $\left(80 \mathrm{~kg} \mathrm{ha}^{-1}\right)$ e uma TMN igual a $30 \%$. No segundo ano, L1 e L2 foram de 10,2 e 20,5 $\mathrm{Mg} \mathrm{ha}^{-1}$ de lodo (massa seca), respectivamente. Nos anos subsequentes, as doses foram padronizadas em 10 e 20 $\mathrm{Mg} \mathrm{ha}^{-1}$ (De Maria et al., 2010).

Em L0, além dos $80 \mathrm{~kg} \mathrm{ha}^{-1}$ de $\mathrm{N}$ utilizados por ano, foram aplicados 21 e $12 \mathrm{~kg} \mathrm{ha}^{-1}$ por ano de $\mathrm{P}_{2} \mathrm{O}_{5}$ e $\mathrm{K}_{2} \mathrm{O}$, respectivamente. A fonte de $\mathrm{N}$ foi a ureia, na adubação de semeadura do milho, e o nitrato ou o sulfato de amônio na cobertura. As fontes de $\mathrm{P}$ e $\mathrm{K}$ foram, respectivamente, superfosfato triplo e cloreto de potássio, aplicados na semeadura.

Nos tratamentos com lodo, utilizou-se complementação mineral com $12 \mathrm{~kg} \mathrm{ha}^{-1}$ por ano de $\mathrm{K}_{2} \mathrm{O}$ via $\mathrm{KCl}$, aplicados na semeadura do milho.

O lodo utilizado foi proveniente da estação de tratamento de esgoto de Jundiaí, SP. A origem do esgoto é predominantemente domiciliar e o tratamento é realizado por meio de lagoas aeradas de mistura completa, seguidas de lagoas de decantação. O condicionamento é feito com polímero sintético.

Alguns atributos químicos dos lodos aplicados na área experimental são apresentados na Tabela 1.

A amostragem do solo foi realizada na camada 0-20 cm, em setembro de 2008, isto é, cerca de um ano após a última aplicação de lodo ou de fertilizante mineral. As amostras foram coletadas em 12 pontos por parcela (quatro repetições por tratamento), com auxílio de trado. As amostras compostas, representativas de cada histórico de uso (L0, L1 e L2) do lodo de esgoto, foram secas ao ar, homogeneizadas e peneiradas em malha de $2 \mathrm{~mm}$, antes da caracterização química, para fins de fertilidade (Tabela 2).

Os experimentos em laboratório foram montados para avaliar a dinâmica de mineralização do $\mathrm{C}$ e do $\mathrm{N}$ no solo, em função dos históricos (L0, L1 e L2) e do $\mathrm{C}$ e do $\mathrm{N}$ adicionados via dose recente de lodo. Para tanto, foram aplicadas novas doses de lodo de esgoto da ETE de Jundiaí (C orgânico, $246 \mathrm{~g} \mathrm{~kg}^{-1}$; N Kjeldahl, $31,9 \mathrm{~g} \mathrm{~kg}^{-1} ; \mathrm{N}^{-\mathrm{NO}_{3}}, 52,3 \mathrm{mg} \mathrm{kg}^{-1} ; \mathrm{N}^{-} \mathrm{NH}_{4}^{+}, 696 \mathrm{mg} \mathrm{kg}^{-1}$ em base seca; e pH 5,6), em L0, L1 e L2. As novas doses foram correspondentes à aplicação de 0,60 e $120 \mathrm{mg} \mathrm{kg}^{-1}$ de $\mathrm{N}$ (D0, D1 e D2, respectivamente), ou 0, 463 e $926 \mathrm{mg} \mathrm{kg}^{-1}$ de C. Dessa forma, as doses D1 e D2 foram equivalentes a 3,8 e $7,6 \mathrm{Mg} \mathrm{ha}^{-1}$ de lodo em base seca ou 120 e $240 \mathrm{~kg} \mathrm{ha}^{-1}$ de N.

Utilizou-se o delineamento experimental inteiramente casualizado, em arranjo fatorial $3 \times 3$, com três repetições.

A mineralização do carbono foi avaliada em $300 \mathrm{~g}$ de solo. As amostras de solo, provenientes de L0, L1 e L2, adicionadas das novas doses de lodo (D0, D1 e D2), foram incubadas em potes herméticos de vidro (1,5 L) por 176 dias, no escuro, a $28 \pm 1^{\circ} \mathrm{C}$, com umidade correspondente a $60 \%$ da capacidade de retenção de água do solo.

Tabela 1. Valores médios ${ }^{(1)}$ de alguns atributos químicos do lodo de esgoto aplicado na área experimental entre 2001 e 2007.

\begin{tabular}{lcc}
\hline Atributo & Média & Erro-padrão \\
\hline pH em água & 5,5 & 1,2 \\
Umidade $\left(\mathrm{g} \mathrm{kg}^{-1}\right)$ & 56,7 & 11,4 \\
Carbono $\left(\mathrm{g} \mathrm{kg}^{-1}\right)$ & 279 & 16,2 \\
Nitrogênio $\left(\mathrm{g} \mathrm{kg}^{-1}\right)$ & 29,3 & 0,8 \\
Fósforo $\left(\mathrm{g} \mathrm{kg}^{-1}\right)$ & 8,1 & 1,0 \\
Potássio $\left(\mathrm{g} \mathrm{kg}^{-1}\right)$ & 1,1 & 0,1 \\
Cálcio $\left(\mathrm{g} \mathrm{kg}^{-1}\right)$ & 14,7 & 4,2 \\
Magnésio $\left(\mathrm{g} \mathrm{kg}^{-1}\right)$ & 1,7 & 0,1 \\
Enxofre $\left(\mathrm{g} \mathrm{kg}^{-1}\right)$ & 19,9 & 2,3 \\
\hline
\end{tabular}

${ }^{(1)}$ Valores médios calculados com base em Bueno et al. (2011).

Tabela 2. Atributos $^{(1)}$ de fertilidade do solo da área experimental.

\begin{tabular}{|c|c|c|c|c|c|c|c|c|c|c|}
\hline Histórico $^{(2)}$ & $\begin{array}{c}\mathrm{pH} \\
\mathrm{CaCl}_{2}\end{array}$ & $\begin{array}{c}\mathrm{MO} \\
\left(\mathrm{g} \mathrm{dm}^{-3}\right)\end{array}$ & $\begin{array}{c}\mathrm{P} \\
\left(\mathrm{mg} \mathrm{dm}^{-3}\right)\end{array}$ & $\mathrm{K}$ & $\mathrm{Ca}$ & $\begin{array}{l}\mathrm{Mg} \\
---(\mathrm{n}\end{array}$ & $\begin{array}{l}\mathrm{H}+\mathrm{Al} \\
\left.\mathrm{dm}^{-3}\right)--\end{array}$ & S & CTC & $\begin{array}{c}\mathrm{V} \\
(\%)\end{array}$ \\
\hline L0 & 5,4 & 26 & 20 & 2,7 & 30 & 17 & 25 & 49,7 & 74,7 & 67 \\
\hline L1 & 4,6 & 30 & 31 & 2,2 & 25 & 11 & 52 & 38,2 & 90,4 & 42 \\
\hline L2 & 4,0 & 36 & 37 & 2,0 & 14 & 4 & 88 & 20,0 & 108,4 & 18 \\
\hline
\end{tabular}

${ }^{(1)}$ Determinados conforme Raij et al. (2001). ${ }^{(2)}$ Históricos de uso de lodo de esgoto entre 2001 e 2007: L0, aplicação de fertilizante mineral nitrogenado ( $80 \mathrm{~kg} \mathrm{ha}^{-1}$ por ano); L1, aplicação de $80 \mathrm{~kg} \mathrm{ha}^{-1}$ por ano de $\mathrm{N}$ via lodo de esgoto (aproximadamente $10 \mathrm{Mg}$ ha-1 por ano, base seca); e L2, aplicação de $160 \mathrm{~kg} \mathrm{ha}^{-1}$ por ano de $\mathrm{N}$ via lodo de esgoto (aproximadamente $20 \mathrm{Mg} \mathrm{ha}^{-1}$ por ano, base seca). S, soma de bases; CTC, capacidade de troca catiônica; V, saturação por bases. 
O lodo das novas doses foi aplicado com umidade original e devidamente homogeneizado com o solo, tendo-se ajustado a umidade posteriormente.

$\mathrm{O}$ carbono liberado na forma de $\mathrm{CO}_{2}\left(\mathrm{C}-\mathrm{CO}_{2}\right)$ foi quantificado conforme Coscione \& Andrade (2006), com captura do $\mathrm{CO}_{2}$ em solução padronizada de $\mathrm{NaOH}$, a $0,5 \mathrm{~mol} \mathrm{~L}^{-1}$ nos primeiros 30 dias de incubação, e, posteriormente, a $0,25 \mathrm{~mol} \mathrm{~L}^{-1}$. A solução de $\mathrm{NaOH}$ contida no interior dos potes herméticos foi periodicamente trocada, e a quantificação do $\mathrm{C}-\mathrm{CO}_{2}$ foi feita imediatamente nas soluções retiradas, por meio de leitura da condutividade elétrica da solução (Rodella \& Saboya, 1999).

Como controle às novas doses, utilizou-se a glicose, aplicada às amostras de L0, L1 e L2 em dose de carbono igual à utilizada em D1 (463 mg kg-1).

A mineralização do $\mathrm{N}$ foi avaliada com as mesmas condições de incubação (temperatura, umidade, escuro) do estudo da degradação do carbono. Amostras de $100 \mathrm{~g}$ dos solos resultantes da combinação entre tratamentos e novas doses foram acondicionadas em frascos de polietileno com capacidade para $0,3 \mathrm{~L}$, com tampas dotadas de pequenos orifícios para permitir trocas gasosas. O número total de potes para incubação foi calculado de modo a permitir a determinação do

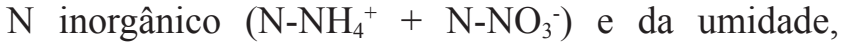
imediatamente após a montagem do experimento (tempo zero) e aos 7, 14, 28, 42, 56, 70, 84, 98, 112 e 126 dias de incubação, com uso de três repetições para cada tratamento.

A extração do $\mathrm{N}$ inorgânico foi feita com solução $2 \mathrm{~mol} \mathrm{~L}^{-1}$ de $\mathrm{KCl}$, e a determinação do teor de $\mathrm{N}$ inorgânico no extrato foi realizada por meio de destilação a vapor, com $\mathrm{MgO}$ e liga de Devarda. O N destilado foi recebido em solução $2 \%$ de $\mathrm{H}_{3} \mathrm{BO}_{3}$, que posteriormente foi titulada com solução padronizada de $\mathrm{H}_{2} \mathrm{SO}_{4}$, conforme Cantarella \& Trivelin (2001).

A umidade gravimétrica do solo foi determinada em outra subamostra dos tratamentos, com vistas à correção dos resultados para massa de matéria seca.

Os dados de mineralização do $\mathrm{C}$ e do $\mathrm{N}$ no tempo foram ajustados à equação de cinética química de primeira ordem: mineralizado $=$ mineralizável $\mathrm{x}$ $\left(1-\mathrm{e}^{-\mathrm{kt}}\right)$, em que mineralizado é a quantidade $\left(\mathrm{mg} \mathrm{kg}^{-1}\right)$ de carbono ou nitrogênio mineralizado no tempo $t$, mineralizável é a quantidade $\left(\mathrm{mg} \mathrm{kg}^{-1}\right)$ de carbono $\left(\mathrm{C}_{0}\right)$ ou nitrogênio $\left(\mathrm{N}_{0}\right)$ potencialmente mineralizável no período de incubação, $\mathrm{k}$ é a constante de velocidade (por dia) da reação de mineralização do carbono $\left(\mathrm{k}_{\mathrm{C}}\right)$ ou do nitrogênio $\left(\mathrm{k}_{\mathrm{N}}\right)$ e t é o tempo de incubação (dias).

Taxas de mineralização do carbono (TMC) e do nitrogênio (TMN) foram calculadas para as doses D1 e D2, em cada histórico de uso do lodo, com uso dos valores de $\mathrm{C}$ ou $\mathrm{N}$ aplicados ao solo e de $\mathrm{C}_{0}$ ou $\mathrm{N}_{0}$, descontados do valor do controle sem nova dose do resíduo $\left(\mathrm{D}_{0}\right)$, de acordo com a seguinte equação: taxa de mineralização $(\%)=100 \mathrm{x}\left(\right.$ mineralizado $\left.-\mathrm{D}_{0}\right) /$ adicionado, em que "mineralizado - $\mathrm{D}_{0}$ " refere-se à diferença entre o $\mathrm{C}_{0}$ da amostra tratada com nova dose de lodo (D1 ou D2) e o $\mathrm{C}_{0}$ do controle que não recebeu nova adição de lodo $\left(\mathrm{D}_{0}\right)$; e adicionado refere-se ao carbono ou nitrogênio recém adicionado ao solo via as doses D1 e D2.

A análise de variância foi utilizada para verificar os efeitos do histórico de uso anterior do lodo e o da aplicação da dose recente, sobre os valores de $\mathrm{C}_{0}, \mathrm{k}_{\mathrm{C}}$, $\mathrm{N}_{0}, \mathrm{k}_{\mathrm{N}}$ e os das taxas de mineralização. A comparação de médias, no caso de $\mathrm{F}$ significativo para algum dos fatores testados, foi realizada pelo teste de Tukey, a 5\% de probabilidade.

\section{Resultados e Discussão}

A aplicação das novas doses do lodo estimulou a liberação de $\mathrm{C}-\mathrm{CO}_{2}$ pelo solo do tratamento $\mathrm{L} 0$, notadamente a partir do vigésimo dia de incubação (Figura 1). Em média, as doses D1 e D2 aplicadas nesse tratamento aumentaram em $15 \%$ os valores de $\mathrm{C}-\mathrm{CO}_{2}$ liberado, em comparação a $\mathrm{D} 0$, entre os 20 e os 170 dias de incubação. Já nos solos com uso anterior de lodo (L1 e L2), as novas doses (D1 e D2) proporcionaram liberação de $\mathrm{C}-\mathrm{CO}_{2}$, em média, apenas $4 \%$ maior do que a observada em D0, entre os 20 e os 170 dias de incubação. Portanto, verificou-se que as novas doses tiveram menor efeito na degradação dos compostos orgânicos nos solos com histórico de uso de lodo.

Para a glicose, não houve efeito dos históricos de uso de lodo ou do fertilizante mineral nitrogenado sobre a degradação do carbono, com degradação média de $76 \%$ do carbono adicionado, aos 170 dias de incubação. Portanto, o uso prévio de lodo de esgoto não prejudicou a degradação da fonte prontamente disponível de $\mathrm{C}$ (glicose).

Além das quantidades liberadas de $\mathrm{C}-\mathrm{CO}_{2}$, as novas doses de lodo também afetaram a dinâmica de mineralização do $\mathrm{C}$ no solo $\left(\mathrm{C}_{0}, \mathrm{k}_{\mathrm{C}}\right.$ e TMC); porém, não 
foi observada interação significativa com os históricos de uso do resíduo (Tabela 3 ). $\mathrm{O}$ valor de $\mathrm{C}_{0}$ foi, em média, $644 \mathrm{mg} \mathrm{kg}^{-1}$ maior nos históricos L1 e L2, em comparação a L0, o que equivale a um fluxo adicional
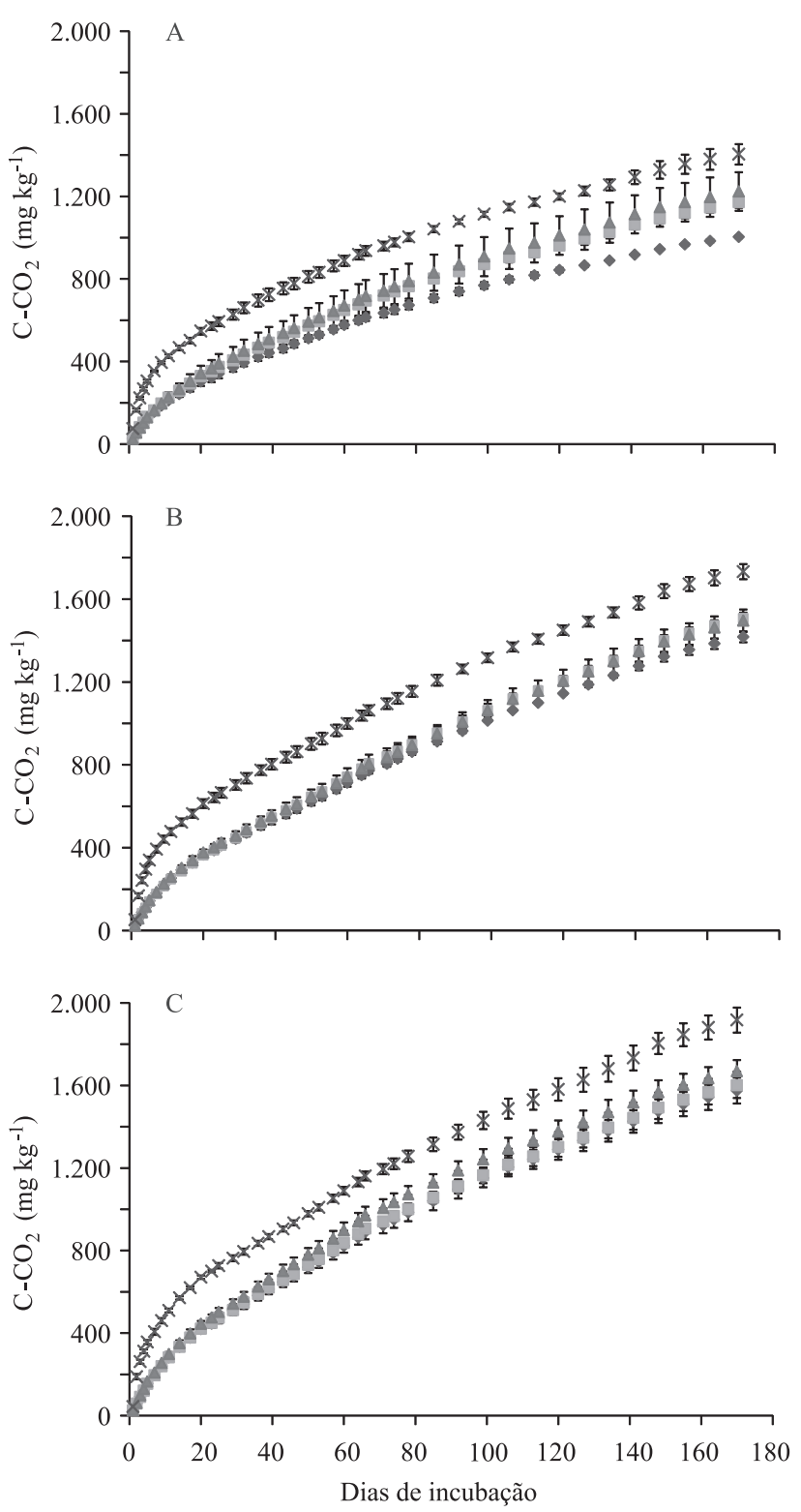

- D0 $\quad \mathrm{D} 1 \quad \Delta \mathrm{D} 2 \quad \times \mathrm{G}$

Figura 1. Valores médios de carbono liberado na forma de $\mathrm{CO}_{2}$ em função do tempo de incubação e das novas doses de lodo - D0, sem adição de lodo; D1, $463 \mathrm{mg} \mathrm{kg}^{-1}$ de C (3,8 $\left.\mathrm{Mg} \mathrm{ha}^{-1}\right)$; e D2, $926 \mathrm{mg} \mathrm{kg}^{-1}$ de C (7,6 Mg ha-1) - ou de glicose $(\mathrm{G}$, dose equivalente a $\mathrm{D} 1)$ adicionadas, em solos com diferentes históricos de uso do lodo entre 2001 e 2007: $\mathrm{A}, 80 \mathrm{~kg} \mathrm{ha}^{-1}$ por ano de $\mathrm{N}$ mineral (L0); $\mathrm{B}, 10 \mathrm{Mg} \mathrm{ha}^{-1}$ por ano de lodo (L1); e C, $20 \mathrm{Mg} \mathrm{ha}^{-1}$ por ano de lodo (L2). de $1,3 \mathrm{Mg} \mathrm{ha}^{-1}$ de $\mathrm{C}-\mathrm{CO}_{2}$, na camada de $0-20 \mathrm{~cm}$, ao se considerar a densidade do solo igual a $1 \mathrm{~g} \mathrm{~cm}^{-3}$.

Pitombo (2011) estimou, a partir de medidas de campo na mesma área experimental do presente trabalho, os fluxos anuais de $\mathrm{C}-\mathrm{CO}_{2}$ em 4,9, 7,3, e $8,7 \mathrm{Mg} \mathrm{ha}^{-1}$ por ano, nos tratamentos L0, L1 e L2, respectivamente. Esses fluxos corrigidos para 170 dias seriam iguais a 2,3, 3,4 e 4,0 $\mathrm{Mg} \mathrm{ha}^{-1}$, ou seja, em média, $1,4 \mathrm{Mg} \mathrm{ha}^{-1}$ de $\mathrm{C}-\mathrm{CO}_{2}$ liberados a mais, nos tratamentos com histórico de uso de lodo. As maiores emissões de $\mathrm{C}-\mathrm{CO}_{2}$ observadas pelos autores nesses tratamentos foram atribuídas ao aumento nos estoques de $\mathrm{C}$ no solo, de $12 \mathrm{Mg} \mathrm{ha}^{-1}$ em L1 e $22 \mathrm{Mg} \mathrm{ha}^{-1}$ em L2. Jin et al. (2011) também verificaram aumento de $10 \mathrm{Mg} \mathrm{ha}^{-1}$ no estoque de $\mathrm{C}$, na camada $0-10 \mathrm{~cm}$, de solo que recebeu, por oito anos, a dose de $22 \mathrm{Mg} \mathrm{ha}^{-1}$ por ano de lodo de esgoto. De forma concomitante ao aumento no estoque de $\mathrm{C}$, os autores relataram maior potencial de liberação de $\mathrm{C}-\mathrm{CO}_{2}$ (aumento de $375 \mathrm{mg} \mathrm{kg}^{-1}$, ou $0,7 \mathrm{Mg} \mathrm{ha}^{-1}$, em comparação ao solo controle). Esses resultados, assim como os obtidos no presente trabalho, evidenciam que parte do $\mathrm{C}$ adicionado via resíduo acumula-se no sistema e passa a ser degradado mais lentamente após sucessivas aplicações do lodo de esgoto.

Em média, o valor de $\mathrm{C}_{0}$ nos tratamentos com novas aplicações de lodo superou em $161 \mathrm{mg} \mathrm{kg}^{-1}$ de $\mathrm{C}-\mathrm{CO}_{2} \mathrm{o}$ valor verificado no solo sem nova aplicação, independentemente do histórico. Desse modo, podese perceber que o histórico de uso de lodo (acréscimo de $644 \mathrm{mg} \mathrm{kg}^{-1}$ ) teve maior influência sobre $C_{0}$ do que as novas aplicações do resíduo (acréscimo de $161 \mathrm{mg} \mathrm{kg}^{-1}$ ), o que é indicativo de que áreas tratadas por médio-longo prazos com lodo podem exibir diferenças fundamentais na dinâmica do carbono e serem menos influenciadas por aplicações recentes.

A velocidade de mineralização do $\mathrm{C}$ somente foi afetada pelo histórico de uso de lodo (Tabela 3), com diferença significativa entre os valores de $\mathrm{k}_{\mathrm{C}}$ de $\mathrm{L} 1 \mathrm{e}$ os de L0 e L2. A redução do $\mathrm{k}_{\mathrm{C}}$ pelo aporte de carbono via lodo de esgoto é atribuída à perda de eficiência da comunidade microbiana (Andrade et al., 2006). Essa explicação parece razoável, se forem considerados os aumentos dos estoques de $\mathrm{C}$ observados na área experimental (Pitombo, 2011) e os valores de $\mathrm{C}_{0}$. A TMC diminuiu cerca de $24 \%$ no solo de L2, em comparação a $\mathrm{L} 0$ e a $\mathrm{L} 1$, que não diferiram entre si. A menor degradação aparente do $\mathrm{C}$ proveniente do lodo recém-adicionado ao solo, no histórico L2, parece contraditória aos elevados valores de $\mathrm{C}_{0}$ 
desse tratamento. No entanto, deve-se ressaltar que os valores de $\mathrm{C}_{0}$ dos tratamentos sem nova aplicação de lodo também foram altos e que também foram usados no cálculo da TMC. Na comparação entre as novas doses de lodo aplicadas, independentemente do histórico de uso de lodo, a TMC para D1 foi de 33\%, enquanto para D2, foi de 19\%. A menor TMC obtida com o incremento da dose de lodo recém aplicada deveu-se, provavelmente, ao aumento da carga orgânica adicionada, que teria suplantado a capacidade microbiana de degradação (Wong et al., 1998). Outra possibilidade refere-se à ocorrência de condição de anaeróbia transitória e localizada no solo, como resposta ao excessivo aumento da taxa respiratória da comunidade microbiana edáfica, em razão do elevado aporte de formas lábeis de $\mathrm{C}$ com o aumento da dose do resíduo (Khalil et al., 2002).

A mineralização do nitrogênio ocorreu com maior intensidade no período inicial de incubação: aos 28 dias nos solos L0 e L1, e um pouco além em L2 (Figura 2). $\mathrm{O}$ solo com aplicação recente de lodo apresentou

Tabela 3. Carbono potencialmente mineralizável $\left(\mathrm{C}_{0}\right)$, constante de velocidade da reação $\left(\mathrm{k}_{\mathrm{C}}\right)$ e taxa de mineralização do carbono (TMC) de acordo com as novas doses de lodo de esgoto adicionadas a solos com diferentes históricos de adição do resíduo ${ }^{(1)}$.

\begin{tabular}{|c|c|c|c|c|}
\hline \multirow[t]{2}{*}{$\operatorname{Dose}^{(2)}$} & \multicolumn{3}{|c|}{ Histórico $^{(3)}$} & \multirow[t]{2}{*}{ Média } \\
\hline & L0 & L1 & L2 & \\
\hline & \multicolumn{3}{|c|}{ Carbono potencialmente mineralizável $\left(\mathrm{mg} \mathrm{kg}^{-1}\right)$} & \\
\hline D0 & 1.066 & 1.746 & 1.866 & $1.559 \mathrm{~B}$ \\
\hline D1 & 1.284 & 1.889 & 1.892 & $1.708 \mathrm{~A}$ \\
\hline D2 & 1.362 & 1.948 & 1.945 & $1.732 \mathrm{~A}$ \\
\hline Média & $1.237 \mathrm{~b}^{(3)}$ & $1.861 \mathrm{a}$ & $1.901 \mathrm{a}$ & - \\
\hline \multirow[t]{2}{*}{ CV $(\%)$} & & 5,15 & & \\
\hline & \multicolumn{3}{|c|}{ Constante de velocidade da reação (por dia) } & \\
\hline D0 & 0,0137 & 0,0092 & 0,0101 & $0,0110 \mathrm{~A}$ \\
\hline D1 & 0,0122 & 0,0083 & 0,0120 & 0,0108A \\
\hline D2 & 0,0117 & 0,0087 & 0,0107 & $0,0104 \mathrm{~A}$ \\
\hline Média & $0,0125 \mathrm{a}$ & $0,0087 \mathrm{~b}$ & $0,0109 \mathrm{a}$ & - \\
\hline \multirow[t]{2}{*}{ CV (\%) } & & 13,38 & & \\
\hline & Taxa de $n$ & zação do & o (\%) & \\
\hline D1 & 47,29 & 43,52 & 9,74 & $33,52 \mathrm{~A}$ \\
\hline D2 & 31,98 & 15,42 & 10,38 & $19,27 \mathrm{~B}$ \\
\hline Média & $39,63 \mathrm{a}$ & $29,47 a$ & $10,06 \mathrm{~b}$ & \\
\hline CV $(\%)$ & & 46,43 & & \\
\hline
\end{tabular}

(1)Médias seguidas de letras iguais, minúsculas nas linhas e maiúsculas nas colunas, não diferem pelo teste de Tukey, a $5 \%$ de probabilidade. ${ }^{(2)}$ Doses de lodo incubadas em laboratório: D0, sem adição de lodo; D1, $463 \mathrm{mg} \mathrm{kg}^{-1}$ de C (3,8 $\left.\mathrm{Mg} \mathrm{ha}^{-1}\right)$; e D2, $926 \mathrm{mg} \mathrm{kg}^{-1}$ de C (7,6 Mg ha-1). ${ }^{(3)} \mathrm{De} 2001$ a 2007: $\mathrm{L} 0,80 \mathrm{~kg} \mathrm{ha}^{-1}$ por ano de $\mathrm{N}$ mineral; $\mathrm{L} 1,10 \mathrm{Mg} \mathrm{ha}^{-1}$ por ano de lodo; e L2, $20 \mathrm{Mg} \mathrm{ha}^{-1}$ por ano de lodo. teores de $\mathrm{N}$ inorgânico superiores ao do solo sem nova aplicação, e as diferenças mais expressivas ocorreram
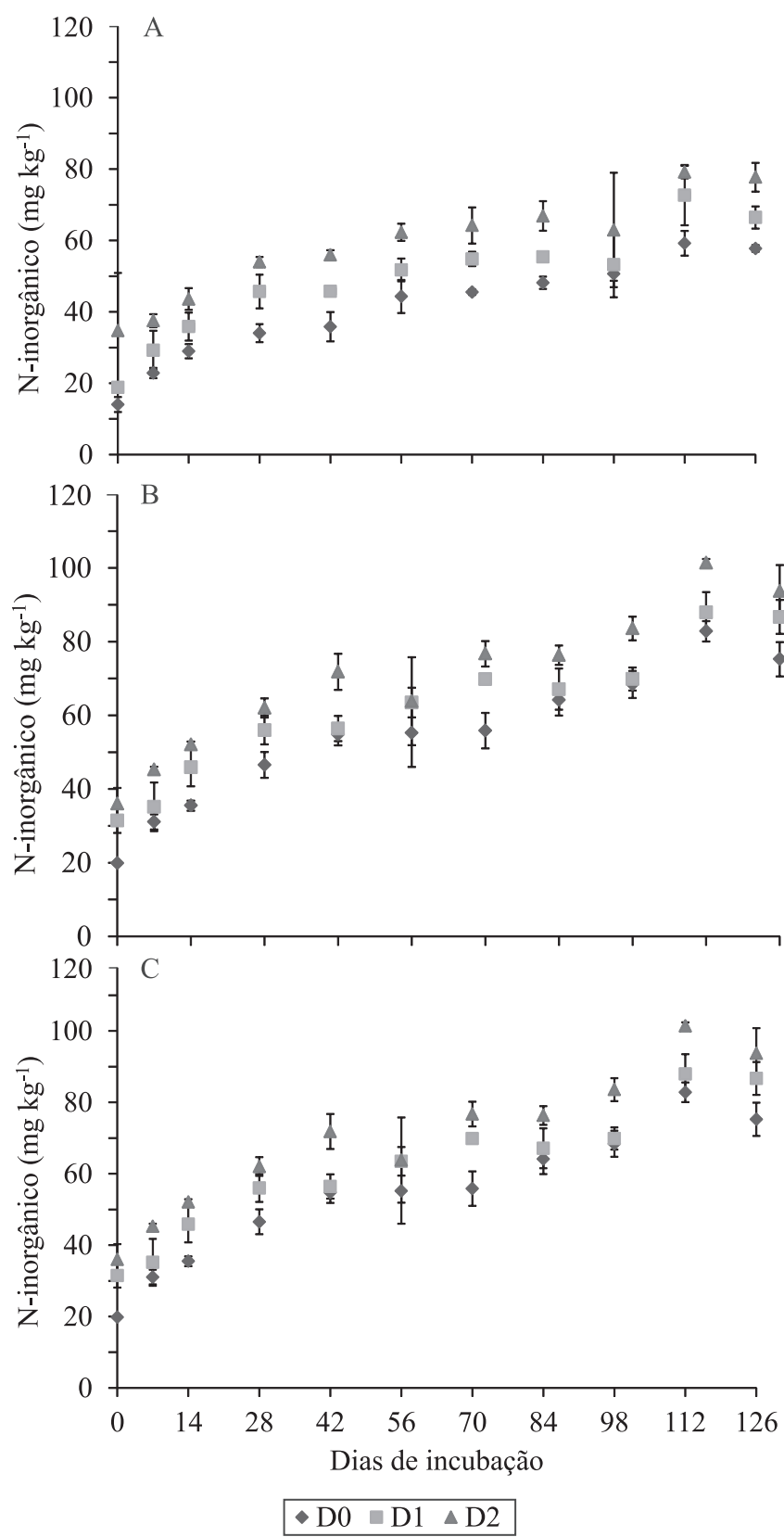

Figura 2. Valores médios de $\mathrm{N}$ inorgânico no solo $\left(\mathrm{NH}_{4}{ }^{+}+\right.$ $\mathrm{NO}_{3}^{-}$) em função do tempo de incubação e das novas doses de lodo - D0, sem adição de lodo; D1, $463 \mathrm{mg} \mathrm{kg}^{-1}$ de C (3,8 $\left.\mathrm{Mg} \mathrm{ha}^{-1}\right)$; e D2, $926 \mathrm{mg} \mathrm{kg}^{-1}$ de C (7,6 $\left.\mathrm{Mg} \mathrm{ha}^{-1}\right)$ - ou de glicose $(\mathrm{G}$, dose equivalente a $\mathrm{D} 1)$ adicionadas, em solos com diferentes históricos de uso do lodo entre 2001 e 2007: A, $80 \mathrm{~kg} \mathrm{ha}^{-1}$ por ano de $\mathrm{N}$ mineral (L0); B, $10 \mathrm{Mg} \mathrm{ha}^{-1}$ por ano de lodo (L1); e C, $20 \mathrm{Mg} \mathrm{ha}^{-1}$ por ano de lodo (L2).

Pesq. agropec. bras., Brasília, v.48, n.5, p.536-544, maio 2013 DOI: 10.1590/S0100-204X2013000500010 
a partir do décimo quarto e do décimo oitavo dias de incubação (Figura 2).

$\mathrm{O}$ solo que nunca havia recebido lodo apresentou a maior diferenciação nos teores de $\mathrm{N}$ entre as doses D0, D1 e D2, o que também foi observado para o carbono. O teor de N inorgânico aumentou, em média, 32\% em D1 e 58\% em D2, na comparação com os teores observados em D0 (período entre o vigésimo oitavo e o centésimo vigésimo sexto dia). Para os solos com histórico de aplicação de lodo, estes aumentos foram de 20 e $41 \%$, em L1, e de 11 e $26 \%$ em L2, respectivamente, para D1 e D2.

O histórico do uso de lodo e a nova aplicação deste resíduo alteraram a dinâmica da disponibilização de $\mathrm{N}$ no sistema de acordo com os parâmetros de cinética química, $\mathrm{N}_{0}$ e $\mathrm{k}_{\mathrm{N}}$ (Tabela 4).

Os solos com aplicações prévias de lodo apresentam potencial em fornecer nitrogênio $\left(\mathrm{N}_{0}\right) 29$ e $64 \%$ maior do que o solo sem aplicação prévia. O fornecimento de $\mathrm{N}$ pela mineralização de compostos orgânicos do solo $\left(\mathrm{N}_{0}\right)$ foi de 92,120 e $151 \mathrm{~kg} \mathrm{ha}^{-1}$ de $\mathrm{N}$, respectivamente,

Tabela 4. Nitrogênio potencialmente mineralizável $\left(\mathrm{N}_{0}\right)$, constante de velocidade da reação $\left(\mathrm{k}_{\mathrm{N}}\right)$ e taxa de mineralização do nitrogênio (TMN) de acordo com as novas doses de lodo de esgoto adicionadas a solos com diferentes históricos de adição do resíduo ${ }^{(1)}$.

\begin{tabular}{|c|c|c|c|c|}
\hline \multirow[t]{2}{*}{$\operatorname{Dose}^{(2)}$} & \multicolumn{3}{|c|}{ Histórico $^{(3)}$} & \multirow[t]{2}{*}{ Média } \\
\hline & L0 & L1 & L2 & \\
\hline \multicolumn{5}{|c|}{ Nitrogênio potencialmente mineralizável $\left(\mathrm{mg} \mathrm{kg}^{-1}\right)$} \\
\hline D0 & 36,64 & 52,99 & 70,23 & $53,19 \mathrm{C}$ \\
\hline D1 & 46,59 & 58,92 & 74,16 & $59,89 \mathrm{~B}$ \\
\hline D2 & 55,71 & 67,65 & 82,49 & $68,62 \mathrm{~A}$ \\
\hline Média & $46,22 \mathrm{c}$ & $59,85 \mathrm{~b}$ & $75,63 a$ & - \\
\hline CV $(\%)$ & & 4,50 & & \\
\hline \multicolumn{5}{|c|}{ Constante de velocidade da reação (por dia) } \\
\hline D0 & 0,0689 & 0,0403 & 0,0408 & $0,0500 \mathrm{~B}$ \\
\hline D1 & 0,0749 & 0,0657 & 0,0585 & $0,0664 \mathrm{AB}$ \\
\hline D2 & 0,0763 & 0,0759 & 0,0688 & $0,0736 \mathrm{~A}$ \\
\hline Média & $0,0734 \mathrm{a}$ & $0,0606 \mathrm{a}$ & $0,0560 \mathrm{a}$ & - \\
\hline CV $(\%)$ & & 26,49 & & \\
\hline \multicolumn{5}{|c|}{ Taxa de mineralização do nitrogênio (\%) } \\
\hline D1 & 17,08 & 9,72 & 4,55 & $10,45 \mathrm{~A}$ \\
\hline D2 & 16,14 & 12,22 & 9,21 & $12,52 \mathrm{~A}$ \\
\hline Média & $16,61 \mathrm{a}$ & $10,97 \mathrm{ab}$ & $6,88 \mathrm{~b}$ & - \\
\hline CV $(\%)$ & & 27,16 & & \\
\hline
\end{tabular}

${ }^{(1)}$ Médias seguidas de letras iguais, minúsculas nas linhas e maiúsculas nas colunas, não diferem pelo teste de Tukey, a $5 \%$ de probabilidade. ${ }^{(2)}$ Doses de lodo incubadas em laboratório: D0, sem adição de lodo; D1, $463 \mathrm{mg} \mathrm{kg}^{-1}$ de C (3,8 $\left.\mathrm{Mg} \mathrm{ha}^{-1}\right)$; e D2, $926 \mathrm{mg} \mathrm{kg}^{-1}$ de C (7,6 $\left.\mathrm{Mg} \mathrm{ha}^{-1}\right) \cdot{ }^{(3)} \mathrm{De} 2001$ a 2007: $\mathrm{L} 0,80 \mathrm{~kg} \mathrm{ha}^{-1}$ por ano de $\mathrm{N}$ mineral; $\mathrm{L} 1,10 \mathrm{Mg} \mathrm{ha}^{-1}$ por ano de lodo; e L2, $20 \mathrm{Mg} \mathrm{ha}^{-1}$ por ano de lodo. para L0, L1 e L2. Portanto, após sete anos de aplicação continuada de lodo de esgoto nessas áreas, observaramse acréscimos de 28 e $59 \mathrm{~kg} \mathrm{ha}^{-1}$ de $\mathrm{N}$, respectivamente, para a dose recomendada de lodo (L1) e o dobro dessa dose (L2). Oliveira et al. (2001) estudaram uma área que recebeu aplicação de lodo de esgoto por dois anos consecutivos e constataram aumento de aproximadamente $40 \%$ no teor de N orgânico, após a primeira aplicação, e de 50\% após a segunda aplicação. Boeira \& Maximiliano (2009) também observaram acúmulo de nitrogênio, em tratamentos que receberam aplicações sucessivas de diferentes lodos de esgoto, por quatro anos.

$\mathrm{O}$ potencial em fornecer nitrogênio $\left(\mathrm{N}_{0}\right)$ do solo que nunca recebeu o resíduo aumentou com as novas doses de lodo aplicadas (6,7 e 15,4 $\mathrm{mg} \mathrm{kg}^{-1}$, respectivamente, para D1 e D2). As novas doses disponibilizaram o equivalente a 13 e $31 \mathrm{~kg} \mathrm{ha}^{-1}$ de $\mathrm{N}$ a mais que o solo sem nova adição de lodo, ao longo de 126 dias de incubação. Jin et al. (2011) encontraram associação entre os valores de $\mathrm{N}$ inorgânico e os de $\mathrm{C}-\mathrm{CO}_{2}$ liberado, em solo com diferentes históricos de uso de lodo, semelhantemente ao verificado no presente trabalho para $\mathrm{C}_{0}$ e $\mathrm{N}_{0}$, que tiveram coeficiente de correlação altamente significativo $\left(\mathrm{r}=0,819^{* *}\right)$. Este resultado possibilita estimar o $\mathrm{N}$ disponível em função da liberação de $\mathrm{C}-\mathrm{CO}_{2}$, o que é relevante, uma vez que a determinação em laboratório do $\mathrm{C}-\mathrm{CO}_{2}$ é mais simples e mais precisa do que a determinação do $\mathrm{N}$ disponível. Para isso, no entanto, é necessário que se determine essa relação em uma ampla gama de condições de uso anterior de lodo e de reaplicações do lodo na mesma área (Andrade et al., 2006).

A velocidade de mineralização não foi alterada pelas aplicações anteriores de lodo (Tabela 4), mas houve alteração com a aplicação das doses recentes, com aumento da velocidade de mineralização na maior dose.

A TMN média de D1 e D2, independentemente do histórico de uso anterior do lodo, foi igual a $12 \%$, valor muito inferior aos 30\% citados na legislação para esse tipo de lodo (Conselho Nacional do Meio Ambiente, 2006). Ao se separarem os resultados de acordo com o histórico, tem-se TMN de $11 \%$, para o solo do tratamento L1, e de 16 e $8 \%$ para L0 e L2, respectivamente. Assim, houve redução na TMN com o uso continuado do lodo de esgoto, o que não significa menor disponibilidade de $\mathrm{N}$ no sistema, pois os valores de $\mathrm{N}_{0}$, conforme discutido anteriormente, foram substancialmente maiores em L1 e L2 do que em L0. 
A partir dos valores de TMN das áreas em questão, e ao se assumir $100 \mathrm{~kg} \mathrm{ha}^{-1}$ como a necessidade em N de uma cultura qualquer, seriam recomendadas doses de lodo de esgoto em base seca (teor de $\mathrm{N}$ no lodo de $32 \mathrm{~g} \mathrm{~kg}^{-1}$ ) iguais a 18, 28 e $45 \mathrm{Mg} \mathrm{ha}^{-1}$, respectivamente, para os históricos L0, L1 e L2. Contudo, se considerados os valores de $\mathrm{N}_{0}$, verifica-se que há maior fornecimento de $\mathrm{N}$ pelo solo após sucessivas aplicações de lodo, o que deve ser acompanhado por redução parcial na dose do resíduo em novas aplicações. Dessa forma, o uso do parâmetro TMN não parece ser uma boa alternativa para o dimensionamento de doses de lodo em áreas que receberam aplicação prévia de lodo de esgoto ou que receberão aplicações sucessivas do resíduo, uma vez que há o risco de se superestimar a dose e aumentar o impacto ambiental da aplicação. Entre as poucas publicações sobre o assunto, pode-se destacar a de Dynia et al. (2006), que avaliaram a lixiviação de nitrato em solo que recebeu cinco aplicações de lodo de esgoto. A perda média total de nitrato por lixiviação no tratamento correspondente à dose recomendada pela legislação foi de $28 \%$ do nitrogênio total aplicado.

Além do uso preferencial do $\mathrm{N}$ potencialmente mineralizável $\left(\mathrm{N}_{0}\right)$ para a recomendação da dose de lodo em solos com histórico de uso anterior do resíduo, outros aspectos devem ser considerados para o aprimoramento do sistema de recomendação desse tipo de resíduo, tais como: a base teórica do cálculo da TMN (Conselho Nacional do Meio Ambiente, 2006), que ignora a mineralização do $\mathrm{N}$ residual proveniente de aplicações anteriores do resíduo no solo; a possibilidade de as novas aplicações de lodo alterarem a mineralização de frações orgânicas residuais parcialmente estabilizadas no solo, o que é um efeito pouco conhecido e de difícil previsão; e a possibilidade de sistemas de produção que usam lodo orgânico se beneficiarem de procedimentos para monitoramento no tempo das mudanças no teor total de $\mathrm{N}$ do solo, como medida auxiliar para a recomendação da dose para reaplicação.

\section{Conclusões}

1. Aplicações sucessivas de lodo de esgoto aumentam o carbono e o nitrogênio potencialmente mineralizáveis no solo.

2. A taxa de mineralização do nitrogênio não é um parâmetro adequado para dimensionar a dose de lodo após sucessivas aplicações do resíduo na mesma área, pois subestima a capacidade do sistema em fornecer $\mathrm{N}$.

\section{Agradecimentos}

Ao Conselho Nacional de Desenvolvimento Científico e Tecnológico (CNPq), pela concessão de bolsa.

\section{Referências}

ANDRADE, C.A. de; BOEIRA, R.C.; PIRES, A.M.M. Nitrogênio presente em lodo de esgoto e a resolução ${ }^{\circ} 375$ do CONAMA. In: COSCIONE, A.R.; NOGUEIRA, T.A.R.; PIRES, A.M.M. (Org.). Uso agrícola de lodo de esgoto: avaliação após resolução $n^{\circ} 375$ do CONAMA. Botucatu: FEPAF, 2010. p.157-170.

ANDRADE, C.A. de; OLIVEIRA, C. de; CERRI, C.C. Cinética de degradação da matéria orgânica de biossólidos após aplicação no solo e relação com a composição química inicial. Bragantia, v.65, p.659-668, 2006. DOI: 10.1590/S0006-87052006000400017.

BETTIOL, W.; CAMARGO, O.A. de. A disposição do lodo de esgoto em solo agrícola. In.: BETTIOL, W.; CAMARGO, O.A. de. (Ed.). Lodo de esgoto: impactos ambientais na agricultura. Jaguariúna: Embrapa Meio Ambiente, 2006. p.25-36.

BOEIRA, R.C. Aspectos experimentais na avaliação da mineralização de nitrogênio de lodos de esgoto incubados com solos. Jaguariúna: Embrapa Meio Ambiente, 2005. 5p. (Embrapa Meio Ambiente. Comunicado técnico, 27).

BOEIRA, R.C.; MAXIMILIANO, V.C.B. Mineralização de compostos nitrogenados após aplicações de lodos de esgoto em quatro cultivos de milho. Revista Brasileira de Ciência do Solo, v.33, p.207-218, 2009. DOI: 10.1590/S0100-06832009000100022.

BOYLE, M.; PAUL, E.A. Carbon and nitrogen mineralization kinetics in soil previously amended with sewage sludge. Soil Science Society of America Journal, v.53, p.99-103, 1989. DOI: 10.2136/sssaj1989.03615995005300010018x.

BUENO, J.R.P.; BERTON, R.S.; SILVEIRA, A.P.D. da; CHIBA, M.K.; ANDRADE, C.A.; DE MARIA, I.C. Chemical and microbiological attributes of an Oxisol treated with successive applications of sewage sludge. Revista Brasileira de Ciência do Solo, v.35, p.1416-1470, 2011. DOI: 10.1590/ S0100-06832011000400040.

CANTARELLA, H.; TRIVELIN, P.C.O. Determinação de nitrogênio inorgânico em solo pelo método da destilação a vapor. In: RAIJ, B. van; ANDRADE, J.C. de; CANTARELLA, H.; QUAGGIO, J.A. (Ed.). Análise química para avaliação da fertilidade de solos tropicais. Campinas: Instituto Agronômico, 2001. p.270-276.

CHIARADIA, J.J.; CHIBA, M.K.; ANDRADE, C.A. de; CARMO, J.B. do; OLIVEIRA, C. de; LAVORENTI, A. $\mathrm{CO}_{2}, \mathrm{CH}_{4}$ and $\mathrm{N}_{2} \mathrm{O}$ fluxes in an Ultisol treated with sewage sludge and cultivated with castor bean. Revista Brasileira de Ciência do Solo, v.33, p.1863-1870, 2009. DOI: 10.1590/S0100-06832009000600035. 
CONSELHO NACIONAL DO MEIO AMBIENTE. Resolução n. ${ }^{\circ}$ 375, de 29 de agosto de 2006. Define critérios e procedimentos, para o uso agrícola de lodos de esgoto gerados em estações de tratamento de esgoto sanitário e seus produtos derivados, e dá outras providências. Diário Oficial [da] República Federativa do Brasil, 30 ago. 2006. Seção 1, p.141-146.

COSCIONE, A.R.; ANDRADE, C.A. Protocolos para avaliação da dinâmica de resíduos orgânicos no solo. In: ANDRADE, J.C. de; ABREU, M.F. de. Análise química de resíduos sólidos para monitoramento e estudos agroambientais. Campinas: Instituto Agronômico, 2006. p.159-177.

DE MARIA, I.C.; CHIBA, M.K.; COSTA, A.; BERTON, R.S. Sewage sludge application to agricultural land as soil physical conditioner. Revista Brasileira de Ciência do Solo, v.34, p.967-974, 2010. DOI: 10.1590/S0100-06832010000300038.

DE MARIA, I.C.; KOCSSI, M.A.; DECHEN, S.C.F. Agregação do solo em área que recebeu lodo de esgoto. Bragantia, v.66, p.291-298, 2007. DOI: 10.1590/S0006-87052007000200013.

DYNIA, J.F.; SOUZA, M.D. de; BOEIRA, R.C. Lixiviação de nitrato em Latossolo cultivado com milho após aplicações sucessivas de lodo de esgoto. Pesquisa Agropecuária Brasileira, v.41, p.855-862, 2006. DOI: 10.1590/S0100-204X2006000500019.

FELIPÓ, M.T.; GARAU, M.A. Comparison of biological and chemical methods to determine available nitrogen in sewage sludge amended soil. Biology and Fertility of Soils, v.5, p.26-30, 1987. DOI: $10.1007 / \mathrm{BF} 00264342$.

FRANCO-HERNÁNDEZ， O.; MCKELLIGAN-GONZALEZ, A.N.; LOPEZ-OLGUIN, A.M.; ESPINOSA-CERON, F.; ESCAMILLA-SILVA, E.; DENDOOVEN, L. Dynamics of carbon, nitrogen and phosphorus in soil amended with irradiated, pasteurized and limed biosolids. Bioresource Technology, v.87, p.93-102, 2003. DOI: 10.1016/S0960-8524(02)00188-8.

GALDOS, M.V.; DE MARIA, I.C.; CAMARGO, O.A. Atributos químicos e produção de milho em um Latossolo Vermelho eutroférrico tratado com lodo de esgoto. Revista Brasileira de Ciência do Solo, v.28, p.569-577, 2004. DOI: 10.1590/ S0100-06832004000300017.

GIL, M.V.; CARBAllo, M.T.; CALVO, L.F. Modelling N mineralization from bovine manure and sewage sludge composts. Bioresource Technology, v.102, p.863-871, 2011. DOI: 10.1016/j. biortech.2010.09.010.

HSEU, Z.Y.; HUANG, C.C. Nitrogen mineralization potentials in three tropical soils treated with biosolids. Chemosphere, v.59, p.447-454, 2005. DOI: 10.1016/j.chemosphere.2004.10.042.

JIN, V.L.; JOHNSON, M.-V.V.; HANEY, R.L.; ARNOLD, J.F. Potential carbon and nitrogen mineralization in soils from a perennial forage production system amended with class B biosolids. Agriculture, Ecosystems and Environment, v.141, p.461-465, 2011. DOI: 10.1016/j.agee.2011.03.016.

KHALIL, M.I.; ROSENANI, A.B.; van CLEEMPUT, O.; BOECKX, P; SHAMAHUDDIN, J.; FAUZIAH, C.I. Nitrous oxide production from an Ultisol of the humid tropics treated with different nitrogen sources and moisture regimes. Biology and Fertility of Soils, v.36, p.59-65, 2002. DOI: 10.1007/s00374-002-0505-1.

LINDEMANN, W.C.; CONNEL, G.; URQUHART, N.S. Previous sludge addition effects on nitrogen mineralization in freshly amended soil. Soil Science Society of America Journal, v.52, p.109-112, 1988. DOI: 10.2136/sssaj1988.036159950052000100 $19 x$.

MEDAILE, L.; BOWDEN, W.B.; SMITH, C.T. Nutrient leaching following land application of aerobically digested municipal sewage sludge in a Northern Hardwood Forest. Journal of Environmental Quality, v.23, p.130-138, 1994. DOI: 10.2134/ jeq1994.00472425002300010021x.

OLIVEIRA, F.C.; MATTIAZZO, M.E.; MARCIANO, C.R.; MORAES, S.O. Lixiviação de nitrato em um Latossolo Amarelo tratado com lodo de esgoto e cultivado com cana-de-açúcar. Scientia Agricola, v.58, p.171-180, 2001. DOI: 10.1590/ S0103-90162001000100026.

PARKER, C.F.; SOMMERS, L.E. Mineralization of nitrogen in sewage sludges. Journal of Environmental Quality, v.12, p.150-156,1983.DOI: 10.2134/jeq1983.00472425001200010028x.

PAUL, K.I.; POLGLASE, P.J.; O'CONNELL, A.M.; CARLYLE, J.C.; SMETHURST, P.J.; KHANNA, P.K. Soil nitrogen availability predictor (SNAP): a simple model for predicting mineralisation of nitrogen in forest soils. Australian Journal of Soil Research, v.40, p.1011-1026, 2002. DOI: 10.1071/SR01114.

PITOMBO, L.M. Estoques de carbono e nitrogênio e fluxos de gases do efeito estufa em solo com diferentes históricos de aplicação de lodo de esgoto. 2011. 61p. Dissertação (Mestrado) Instituto Agronômico, Campinas.

PRATT, C.F.; BROADBENT, F.E.; MARTIN, J.P. Using organic wastes as nitrogen fertilizers. California Agriculture, v.27, p.10-13, 1973.

RAIJ, B. van; ANDRADE, J.C. de; CANTARELLA, H.; QUAGGIO, J.A. (Ed.). Análise química para avaliação da fertilidade de solos tropicais. Campinas: Instituto Agronômico, 2001. 285p.

RODELLA, A.A.; SABOYA, L.V. Calibration for conductimetric determination of carbon dioxide. Soil Biology and Biochemistry, v.31, p.2059-2060, 1999. DOI: 10.1016/S0038-0717(99)00046-2.

SANTOS, H.G. dos; JACOMINE, P.K.T.; ANJOS, L.H.C. dos; OLIVEIRA, V.A. de; OLIVEIRA, J.B. de; COELHO, M.R.; LUMBRERAS, J.F.; CUNHA, T.J.F. (Ed.). Sistema Brasileiro de classificação de solos. 2.ed. Rio de janeiro: Embrapa Solos, 2006. $306 \mathrm{p}$.

UNITED STATES ENVIRONMENTAL PROTECTION AGENCY. 40 CFR - title 40 - protection and environment: part 503 - standards for the use or disposal of sewage sludge. Washington: U.S. EPA, 1993.

WONG, J.W.C.; LAI, M.; FANG, M.; MA, K.K. Effect of sewage sludge amendment on soil microbial activity and nutrient mineralization. Environment International, v.24, p.935-943, 1998. DOI: 10.1016/S0160-4120(98)00075-0.

Recebido em 24 de julho de 2012 e aprovado em 26 de abril de 2013 